We appreciate the response to this publication feature - and weicome all contributions. Contributions may be sent to Phil Oshel, our Technical Editor at:

$\begin{array}{ll}\text { Mr. Phil Oshel } & \text { (608)833-2885 } \\ \text { PO Box 620068 } & \text { eMail: oshel@terracom.net } \\ \text { Middleton W/ } 53562 & \end{array}$

\title{
Visualizing Capillary Beds
}

Standard methods of visualizing capillary beds in tissue often require perfusing the capillaries with colored markers such as India ink or casting materials. The specimen material is subject to substantial artifact from incomplete perfusion and distortion of delicate vascular components due to excessive perfusion pressure.

A simple method to minimize these potential problems uses the blood components in unperfused tissue. The endogenous peroxidase in red blood cells is easily reduced in the presence of hydrogen peroxide with the resultant oxidation of the chromagen 3,3'-diaminobenzidine (DAB) and formation of a dark reaction product throughout the vasculature. Exposing the tissue sections to peroxidase-conjugated $\mathrm{lgG}$ to enhance background staining can further enhance the reaction.

The procedure is as follows:

1) Cool the tissue immediately after euthanizing the animal or removing the organ in order to promote blood clotting.

2) After 1 to 2 hours, cut the tissue into $1 \mathrm{~cm}^{2}$ blocks, immerse in cold $4 \%$ paraformaldehyde in $0.1 \mathrm{M}$ Phosphate buffer $(\mathrm{pH} 7.2$ to 7.4$)$ and fix for 24 to 48 hours at $40^{\circ} \mathrm{C}$.

3) Wash with buffer to remove excess fixative and cut on a vibrating microtome in 50 to $60 \mu \mathrm{m}$ sections.

4) Immerse sections in a $0.02 \%(W / V)$ solution of $3,3^{2}$ diaminobenzidine (DAB) containing $0.03 \% \mathrm{H}_{2} \mathrm{O}_{2}$ for 15 to 30 minutes at room temperature.

5) The reaction may be amplified by incubating the tissue sections in a 1:100 (vol/vol) dilution of peroxidase-conjugated goat anti-rat (or appropriate species depending on your tissue). IgG in $0.02 \mathrm{M}$ phosphate buffer for 30 minutes at room temperature. Wash sections three times for 15 minutes to remove unbound $\operatorname{lgG}$ and react with $\mathrm{DAB}-\mathrm{H}_{2} \mathrm{O}_{2}$ developing solution as above.

DAB- $\mathrm{H} 202$ defined microangiograms can be digitized and vessels quantified using image analysis programs. Three-dimensional reconstruction of serial sections can be produced and parameters such as volume density calculated.

The major source of artifact in this method is shrinkage associated with aldehyde fixation. Shrinkage can be limited by using freshly prepared fixatives and standard block sizes and fixation times. Estimates of shrinkage can then be taken into account when analyzing and quantitating microvasculature.

Additional details and illustrations are found in: Sherman, 9. and W. Paull (1985). New Method for Visualization of Vascular Networks in Nonperfused Fixed Tissues. Stain Technology. 60:2 p.89.

$$
\text { Debby Sherman, Purdue University }
$$

\section{A Note on Solvents for Embedding with Epon}

John Luft originally suggested 1-2 epoxy propane or propylene oxide as an intermediate solvent for epoxy infiltration because he reasoned that its chemical structure would allow it to be incorporated into the epoxy resin.

But I question if this could ever happen. Consider the boiling points of the three solvents which are being compared:

$\begin{array}{ll}\text { ethanol. .......... } & 78.3^{\circ} \mathrm{C} \\ \text { acetone. ........ } & 56^{\circ} \mathrm{C} \\ \text { epoxy propane... } & 34.3^{\circ} \mathrm{C}\end{array}$

Most epoxies are cured at $70^{\circ} \mathrm{C}$ or above. At this temperature acetone and especially epoxy propane will very rapidly evaporate from the mixture. But alcohol will not. Years back, I mixed epoxy resin with ten percent of each solvent, and put the three samples into the curing oven at $70^{\circ} \mathrm{C}$. The acetone an epoxy propane mixtures cured perfectly normally, but the alcohol sample stayed sticky. I think it just doesn't evaporate.

Bottom line: since then my lab has always used acetone as a less hazardous alternative to epoxy propane. They both reduce the viscosity of epoxy resin about the same amount. The historically minded could check my 1968 paper, "viscosity changes in Araldite during polymerization", Laboratory Practice 17:707-708.

Mel Dickson, University of New South Wales, Australia

\section{A Blocking Agent for Endogenous Peroxide}

We have used $0.1 \%$ sodium azide $+3 \%$ hydrogen peroxide for many years without damage to acetone-fixed frozen sections. This blocking protocol will work with almost all slide-based specimens for blocking endogenous peroxidase.

The $0.1 \%$ sodium azide solution is prepared as a stock solution and the hydrogen peroxide is added prior to use (within several hours). Azide stock is stable for 2 months and a $10 \mathrm{X}$ (or $1 \%$ ) solution can be made if large volumes are used.

The following mix is used for 10 minutes at room temperature after rehydrating slides following fixation in acetone:

$0-1 \%$ sodium azide $45 \mathrm{~mL}$

$0.3 \%$ hydrogen peroxide $5 \mathrm{~mL}$

(use $3 \%$ hydrogen peroxide for peripheral blood smears)

Discard azide solutions according to waste management practice at your institution.

This method is adapted from the method in: $\mathrm{Li}_{i}$ C. Y., S.C. Ziesmer, and 0. Lazcano-Villareal. 1987. Use of azide and hydrogen peroxide as an inhibitor for endogenous peroxidase in the immunoperoxidase method. Journal of Histochemistry \& Cytochemistry. 35(12):1457-60.

Timothy Plummer, Mayo Clinic, Rochester. Minnesota

\section{To Obtain Vertical Sections of Cell Monolayers for TEM}

Our cell monolayers were positioned on the very edge of our specimen blocks. Upon sectioning and mounting, the ultra-thin sections folded over, hammering TEM examination of the cell monolayers. The following soda-straw method centered the monolayers on the specimen blocks and solved our problem:

1) Embed the monolayer within the culture dish with Polybed 812.

2) Following heat polynienzation, use a hammer to extract the sample from the dish.

3) Secure the disk-shaped sample in a vise and with a jeweler's or coping 
saw cut out a $2 \mathrm{~mm}^{2}$ piece.

4) Prepare a mounting block by sawing off the pyramidal end of a blank resin block, previously polymerized in a BEEM capsule.

5) Glue the resin piece with the specimen to the prepared surface with cyanoacrylate glue. Orient as desired. Note: unpolymerized resin might work as well, but I have found that cyanoacrylate fastens the specimen in place quickly and surely, and without the sticky mess associated with resin.

6) Insert the block specimen-side up into a new BEEM capsule.

7) Cut the end off of a plastic soda-straw from your favorite fast-food restaurant and place the plastic cylinder around the specimen.

8) Reinforce the specimen by filling the cylinder with resin and polymerizing.

Dorothy Sorenson, Eastern Michigan University

\section{Preparation of Buffy Coat Leukocytes for Transmission Electron Microscopy}

The following procedure is an easy method to prepare buffy coat leukocytes for TEM. The cells are differentially centrifuged, resulting in layers enriched for each cell type. Granulocytes (eosinophils, neutrophils, and basophils) are found in the layer next to erythrocytes at the bottom of the buffy coat, monocytes and large lymphocytes are above the granulocytes, small lymphocytes are next, and platelets are at the top. Fixing the centrifuged cells without disturbing the layers results in a plug that can be processed easily while maintaining the cell layers.

Procedure:

1) Collect blood in standard EDTA tube.

2) Spin blood sample in clinical centrifuge to obtain a visible buffy coat (about $3500 \mathrm{rpm}$ for 20 minutes).

3) Carefully remove just the straw-colored plasma layer with pipette. A small amount of plasma may be left, if necessary, to avoid disturbing the buffy coat.

4) Gently add buffered glutaraldehyde by running it drop-wise down the sides of the tube, again without disturbing the buffy coat layer. $3 \%$ glutaraldehyde in $0.1 \mathrm{M}$ cacodylate or Sorensen's phosphate buffer, $\mathrm{pH} 7.2$ to 7.4 is suitable for primary fixation. Use this buffer in all buffer-only steps below.

5) After about 30 minutes, test the plug to see if it's fixed - it should feel rubbery with gentle prodding. If it is not firm, replace the fixative with fresh fixative and allow the buffy coat to fix for another 15 to 30 minutes.

6) Remove the fixed buffy coat plug from the tube. A small spatula or sharpened wooden applicator stick works well. This step can get a little messy, but the idea is to remove the plug as a coin-shaped disk with only a small number of adherent erythrocytes on the underside. Erythrocytes can be washed off with buffer if needed.

7) Place the plug with the erythrocyte-side down on dental wax and cut out a vertical slice. Take the slice and slice it again in the top-to-bottom direction into tissue-sized blocks ( 1 to $2 \mathrm{~mm}$ thick). Each block will contain all cell types layered as described above.

8) Place the blocks into a processing vial, and fix for an additional hour in glutaraldehyde.

9) Wash in $0.1 \mathrm{M}$ buffer, $2 \times 10$ minutes in each.

10) Post-fix in $1 \%$ osmium tetroxide in $0.1 \mathrm{M}$ buffer for 1 hour.

11) Rinse several times briefly in distilled water.

12) Dehydrate in ethanol: $50 \%, 70 \%$, and $95 \%, 15$ minutes in each.

13) Absolute ethanol: $2 \times 15$ minutes in each.

14) Propylene oxide: $2 \times 15$ minutes in each.

15) Propylene oxide: epoxy resin (1:1) for 1 hour.

16) $100 \%$ resin $-2 \times 8$ hours

17) Orient for embedding so that the largest face of the block will be sectioned (to get all the cell layers in the section).

18) Polymerize blocks as usual (at $60^{\circ} \mathrm{C}$ for 3 days or $90^{\circ} \mathrm{C}$ for 60 to 90 minutes).

Other dehydration and infiltration schedules will most likely work (e. g., acetone dehydration, different resins). Another variation is to remove the buffy coat before fixation and centrifuge it in an equal volume of fixative, thus eliminating platelets and erythrocytes while retaining the layering of leukocytes in embedded blocks (thanks to Ann Lehman at Trinity College, Hartford CT).

Reference: Anderson, D.R. 1965. A method for preparing peripheral leucocytes for electron microscopy. J. Ultrastructure Research 13:263-268.

Jane A. Fagerland, Abbott Laboratories

\section{Making Flexible Micrometer-Sized Needles}

We have used tungsten needles of about one micrometer with our high voltage electron microscope. What we were looking for was a rather rigid "needle point" on which to mount micrometer-sized objects. It was found that when we attempted to move these small specimens around and to get them to stick to the needles, the needles would bent very easily when they contacted the glass slides our specimens were prepared on. So tungsten would make good flexible needles.

We made them by electrolytically etching 5 mil. tungsten wires in $\mathrm{NaNO}_{3}$ solution:

1) Use a $30 \mathrm{~mL}$ beaker with $20 \mathrm{~mL}$ of roughly 0.5 molar $\mathrm{NaNO}_{3}$ with a two inch carbon evaporation electrode (1/8 inch diameter) connected to one side of a small 6.3 VAC (yes, $A C$ ) transformer and placed in the beaker.

2) 5 mil. tungsten wire was held in a small alligator test lead, with the other end connected to the other side of the transformer. The key part of the technique was to hold the alligator clip in hand and dip only the very tip of the tungsten wire into the solution (1/8 inch or less), as observed from below the interface. This had to be held steady for five minutes or so, then check the progress in the light microscope. Two or three iterations would usually suffice.

3) The wire was usually fused into a drawn glass tube for a handle.

Observing the results is the key. Also, once the tip of the wire is bent it would not return all the way to its being straight, which was at times frustrating.

Dave Barnard, New York State Department of Health, Wadsworth Center

A De-embedding Solution for $L X-112$ Resin

We at Ladd sell LX-112 and one procedure we know of that may work for de-embedding specimens in it is prepared as follows:

1) Prepare saturated solution of $\mathrm{KOH}$ in absolute ethanol. Let stand overnight.

2) Pour off supernatant fluid. This is the epoxy solvent.

3) Trim block of excess epoxy resin.

4) Let specimen soak in solvent until resin is removed. Time will vary depending on size of block.

5) Wash with several changes of absolute ethanol.

6) Prepare for SEM

Note: This solution may do damage to your specimen. I suggest trying this procedure on only one or two of your less critical samples.

Havat, M.A. (1989). Principles and Techniques of Electron Microscopy: Biological Applications. 3rd Edition. CRC Press, Inc. Boca Raton, Florida. 